\title{
O MECANISMO DE AUTOPROPULSÃO DE VÓRTICES OCEÂNICOS: UMA REVISÃO
}

\author{
José Luiz Lima de Azevedo ${ }^{1}$ e Mauricio Magalhães Mata² \\ Recebido em 9 setembro, 2009 / Aceito em 27 maio, 2010 \\ Received on September 9, 2009 / Accepted on May 27, 2010
}

\begin{abstract}
Oceanic eddies are effective carriers of momentum, mass, heat, of chemical and biological characteristics associated generally with their place of origin. These features exercise influence on global circulation, in the distribution of large-scale water masses and in the biology of the oceans. This influence does not only involve the transfer of energy and properties associated with the place of origin of the eddy but also their strong performance in mixing processes. The motion of eddies across the oceans are primarily driven by three factors: the self-propulsion which is intrinsic to the feature and moves it towards the west; the advection by others currents and the influence of eddies nearby. This work focuses on the first point, where the self-propulsion of isolated eddies is widely reviewed and discussed. The expression that allows the approximate calculation of the translation speed of isolated vortices is deduced. The basic equations (shallow water, the Bernoulli function and integrated meridional momentum) required for this development are presented and discussed as well as the meridional forces that act on these features in motion, where the mathematical formalism associated with each of them is also reviewed. This review shows that all isolated vortices are self-propelled towards the west, regardless of the hemisphere considered. It also shows that three meridional forces act on the eddies in motion: (1) the $\beta$ force, due to the difference of the Coriolis parameter between the northern and southern hemispheres of the eddy, (2) the Coriolis force and the (3) ambient force, due to the action of the external fluid on the isolated eddy. Several analyses can be made with respect to these forces and this review also presents, as an example, a comparison of the $\beta$ forces acting in anticyclonic and cyclonic eddies, with the same characteristics, moving in the southern hemisphere. It is concluded that the $\beta$ force in the former is greater than the force in the latter. This study also comments aspects related with the balance of forces on particles rotating inside the eddy, where the gradient, geostrophic, quasi-geostrophic and cyclostrophic balances are discussed.
\end{abstract}

Keywords: isolated oceanic eddies, drift of oceanic eddies, self-propulsion, beta $(\beta)$ force, ambient force.

RESUMO. Vórtices oceânicos são efetivos transportadores de momentum, massa, calor, de características químicas e biológicas associadas, em geral, com o seu local de origem. Estas feições exercem influência na circulação global, na distribuição de larga escala de massas d'água e na biologia dos oceanos. Esta influência não envolve somente transferência de energia e de propriedades associadas com o local de origem do vórtice, mas também a sua acentuada atuação em processos de mistura. 0 movimento dos vórtices pelos oceanos é influenciado basicamente por três fatores: a autopropulsão intrínseca à feição, que a impulsiona no sentido oeste; a advecção por correntes externas e a influência de vórtices próximos. Para um melhor entendimento da importância dos vórtices nas largas escalas o seu comportamento isolado deve ser estudado. Este trabalho se concentra no primeiro aspecto, onde a autopropulsão de vórtices isolados é amplamente revisada e discutida. A expressão que permite o cálculo aproximado da velocidade de translação de vórtices isolados é deduzida. As equações básicas (águas rasas, função de Bernoulli e integrada do momentum meridional) necessárias para este desenvolvimento são apresentadas e discutidas, assim como as forças meridionais que atuam sobre estas feições em movimento, onde o formalismo matemático associado a cada uma delas é também revisado. Esta revisão mostra que todos os vórtices isolados são autopropulsionados no sentido oeste, independentemente do hemisfério considerado. Mostra ainda que três forças meridionais atuam sobre os vórtices em movimento: (1) a força $\beta$, devida à diferença do parâmetro de Coriolis entre os hemisférios norte e sul do vórtice, (2) a força de Coriolis e a (3) força ambiente, devida à ação do ambiente externo sobre 0 vórtice. Diversas análises podem ser feitas com respeito a estas forças e esta revisão também apresenta, como exemplo, uma comparação da força $\beta$ que atua na situação de vórtices anticiclônicos e ciclônicos, de mesmas características, em trânsito no hemisfério Sul. Conclui-se que a força $\beta$ do primeiro é superior a do segundo. 0 presente estudo aborda ainda aspectos relacionados com o balanço de forças sobre as partículas em rotação dentro de um vórtice, onde os balanços gradiente, geostrófico, quase-geostrófico e ciclostrófico são discutidos.

Palavras-chave: vórtices oceânicos isolados, deriva de vórtices oceânicos, autopropulsão, força beta $(\beta)$, força ambiente.

\footnotetext{
${ }^{1}$ Universidade Federal do Rio Grande - FURG, Instituto de Oceanografia, Laboratório de Estudos dos Oceanos e Clima, Rua Eng. Alfredo Huch, 475, Centro, 96211-900 Rio Grande, RS, Brasil. Tel.: (53) 3233-6880; Fax: (53) 3233-6652 - E-mail: joseazevedo@furg.br

2Universidade Federal do Rio Grande - FURG, Instituto de Oceanografia, Laboratório de Estudos dos Oceanos e Clima, Rua Eng. Alfredo Huch, 475, Centro, 96211-900 Rio Grande, RS, Brasil. Tel.: (53) 3233-6879; Fax: (53) 3233-6652 - E-mail: mauricio.mata@furg.br
} 


\section{INTRODUÇÃo}

Vórtices isolados são feições oceânicas caracterizadas por linhas de corrente fechadas e que possuem fluxos estruturados associados com uma massa isolada de água com características anômalas (mais especificamente quanto à temperatura e salinidade) daquelas que circundam a feição (Flierl, 1979). Enquanto as ondas são efetivas transportadoras de energia, os vórtices transportam energia e massa através dos oceanos (e.g., Olson, 1991). Com maior rigor, os vórtices transportam momentum, calor, massa e características químicas e biológicas das águas de seu ponto de origem, contribuindo na circulação global, na distribuição de larga escala de massas d'água e na biologia dos oceanos (e.g., Robinson, 1983). A influência dos vórtices na circulação oceânica de larga escala envolve, não somente, a transferência de energia e de propriedades associadas com 0 seu local de origem, mas também 0 seu impacto em processos de mistura (e.g., Olson, 1991). No plano- $\beta$ (onde 0 parâmetro de Coriolis varia de acordo com a latitude, tomada com relação a uma latitude de referência, em geral situada no centro do plano), um vórtice isolado é "autopropulsionado" no sentido oeste (e.g., Nof, 1981b; Killworth, 1983; Cushman-Roisin et al., 1990). Esta autopropulsão é intrínseca ao vórtice e seu mecanismo será detalhado ao longo deste estudo. 0 trabalho de Byrne et al. (1995) estuda vórtices provenientes da região de retroflexão da Corrente das Agulhas. A Figura 1, retirada de Byrne (2000), mostra a trajetória de alguns vórtices isolados, provenientes desta região, a partir de dados Geosat. 0 movimento destas feições no sentido oeste é evidenciado na figura. Cabe destacar que estes vórtices estão sujeitos à ação advectiva de correntes e de outros vórtices.

Enquanto 0 vórtice translada no sentido oeste toda a sua anomalia de massa é carregada junto com ele (Nof, 1981b). Caso os efeitos advectivos externos citados acima não sejam considerados, o movimento do vórtice será sempre no sentido oeste apresentando um desvio com relação à linha do Equador que é função das não-linearidades da feição (e.g., Firing \& Beardsley, 1976). A inclinação do fundo também pode induzir movimento nos vórtices. Neste caso ela atua analogamente ao efeito $\beta$ sobre a feição, a qual poderá então se mover mesmo estando no plano- $f$ (neste cenário o parâmetro de Coriolis é mantido constante ao longo do plano, em geral com valor associado à latitude central do referido plano), tal qual uma onda topográfica (e.g., Nof, 1983; Shi \& Nof, 1994). 0 movimento de vórtices para oeste pode ser acompanhado com a utilização de satélites (e.g., Souza, 2005), os quais detectam os vórtices por anomalias no nível do mar (e.g., Hwang et al., 2004; Lentini et al., 2006; Chelton et al., 2007) ou pela diferença de temperatura entre a água interna do vórtice e aquela que 0 circunvizinha (e.g., Souza et al., 2006). 0 estudo de Smith \& O'Brien (1983) recomenda que para um melhor entendimento da importância dos vórtices nas largas escalas o seu comportamento isolado deve ser estudado. 0 objetivo deste trabalho é apresentar uma revisão sobre a autopropulsão para oeste de vórtices isolados, os quais serão representados por uma região de circulação ciclônica ou anticiclônica, delimitada por uma linha de corrente livre, que isola a feição do oceano externo. As forças meridionais que atuam sobre estes vórtices, enquanto em movimento, também serão apresentadas. A revisão é enriquecida com diversas figuras e esquemas que facilitam 0 entendimento do leitor sobre 0 tema. Após esta introdução, a seção 2 apresenta uma revisão teórica das principais equações que são necessárias para que uma expressão que forneça a velocidade de autopropulsão de vórtices isolados possa ser obtida. São destacadas: (1) as equações de águas rasas, (2) a função de Bernoulli e (3) a equação integrada do momentum meridional, onde se destaca que todas elas estão escritas em um sistema móvel de coordenadas instalado no centro do vórtice isolado. A seção 3 apresenta as três forças que atuam meridionalmente sobre o vórtice isolado em movimento, ou seja, (1) a força $\beta$, (2) a força de Coriolis e (3) uma força ambiente, decorrente da ação do fluido externo ao vórtice sobre ele próprio. A seção 4 enfoca a deriva para oeste dos vórtices isolados onde uma expressão que permite o cálculo da velocidade de autopropulsão é apresentada. A seção 5 discorre sobre 0 balanço meridional das forças que atuam sobre o vórtice isolado em movimento. Os movimentos de vórtices ciclônicos e anticiclônicos no hemisfério Sul são analisados nas seções 4 e 5 . Finalmente a seção 6 apresenta uma discussão sobre o tema da autopropulsão de vórtices e forças meridionais envolvidas no movimento das feições no sentido oeste. Os trabalhos de Nof (1981a) e Nof (1983) são referências básicas para esta revisão.

\section{REVISÃO TEÓRICA}

Esta seção se dedica à revisão das equações básicas necessárias para o desenvolvimento de uma expressão que permita o cálculo da velocidade de autopropulsão para oeste de vórtices isolados. Será suposto que: (a) o oceano pode ser descrito por uma camada rasa superior e uma camada inferior infinitamente profunda considerada sem movimento, (b) que o movimento do vórtice isolado é invíscido, estacionário e não-difusivo e (c) que a feição se desloca sem significativas trocas na forma de sua estrutura (Nof, 1983). 


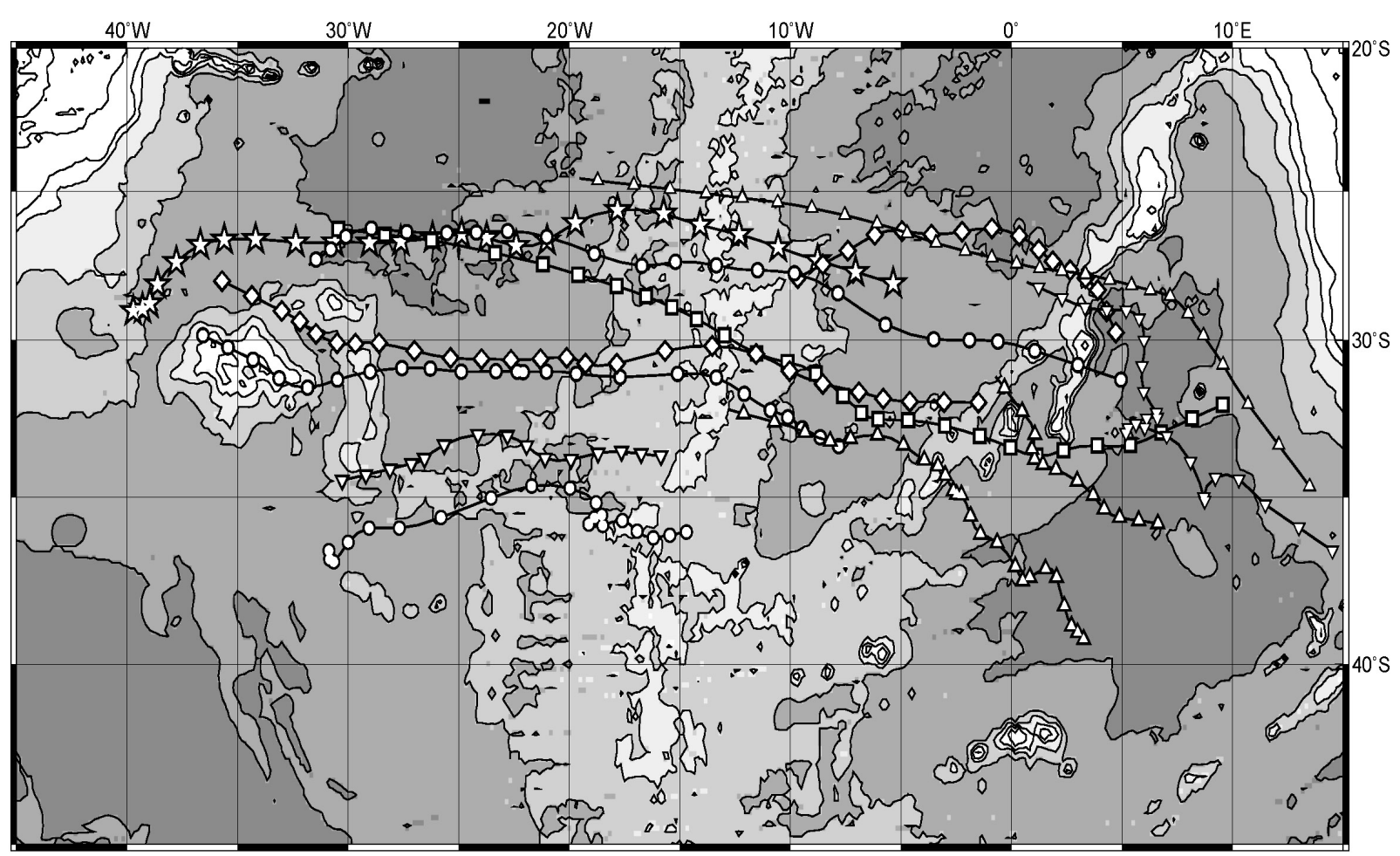

Figura 1 - Trajetórias de vórtices individuais a partir de dados Geosat, conforme estudos de Byrne et al. (1995) e Byrne (2000). A batimetria está contornada com intervalos de 1000 metros. As trajetórias estão suavizadas a partir da estimativa do centro de cada feição. 0 espaçamento temporal entre duas posições sucessivas dos vórtices, nas trajetórias estudadas, é de 34 dias. A figura foi retirada de Byrne (2000).

\subsection{As equações de águas rasas}

Nesta subseção serão deduzidas as equações de águas rasas que descrevem o movimento de um vórtice isolado. Inicialmente será suposto que o vórtice translada na direção leste (sentido positivo do eixo $x$ ) com velocidade $c$. Um sistema móvel de coordenadas está instalado no centro da feição. 0 vórtice isolado será descrito mediante a utilização de um modelo invíscido de duas camadas homogêneas, conforme mostra a Figura 2 (para o caso de um vórtice anticiclônico, i.e., com centro de alta pressão), onde a profundidade $H_{I}$ da camada inferior é infinitamente maior que a profundidade $H_{S}$ da parte não perturbada da camada superior. Esta última camada possui densidade $\rho_{0}$ e consiste do vórtice propriamente dito e da região sobre a termoclina $T C$ (interface entre as camadas) onde esta é plana. A camada abissal, a qual é suposta estagnada, tem densidade $\rho_{0}+\Delta \rho$, onde $\Delta \rho$ é a diferença de densidade entre as duas camadas $\left(\Delta \rho \ll \rho_{0}\right)$. 0 vórtice isolado se move sobre esta camada sem induzir movimentos no fluido que a compõe. No esquema esquerdo da Figura 2 um sistema fixo de coordenadas é mostrado. As variáveis independentes deste sistema são $(X, Y, Z, T)$, que correspondem às coordenadas zonal, meridional e vertical e 0 tempo, respecti- vamente. A Figura 2 mostra ainda 0 deslocamento da superfície livre do mar $\eta(X, Y, T)$ devido à presença do vórtice, o deslocamento da termoclina $\xi(X, Y, T)$ na parte inferior desta feição e a profundidade $h(X, Y, T)$ do vórtice, referenciadas com relação a um sistema de coordenadas fixo na Terra. A variável $\eta$ é considerada positiva no sentido positivo do eixo $Z$ enquanto a variável $\xi$ é considerada positiva em sentido contrário. A geometria do vórtice permite escrever

$$
h=\eta+H_{S}+\xi
$$

As equações invíscidas de águas rasas para um fluido homogêneo e sujeito ao equilíbrio hidrostático, escritas em um sistema de coordenadas fixo na Terra que é composto pelas variáveis independentes $(X, Y, Z, T)$, têm como origem as equações originais do momentum, da continuidade e hidrostática, as quais são apresentadas abaixo (e.g., Gill, 1982; Pedlosky, 1987; CushmanRoisin, 1994):

$$
\begin{aligned}
\frac{\partial U}{\partial T} & +U \frac{\partial U}{\partial X}+V \frac{\partial U}{\partial Y}-\left(f_{0}+\beta Y\right) V \\
& =-\frac{1}{\rho_{0}} \frac{\partial P_{D}}{\partial X},
\end{aligned}
$$



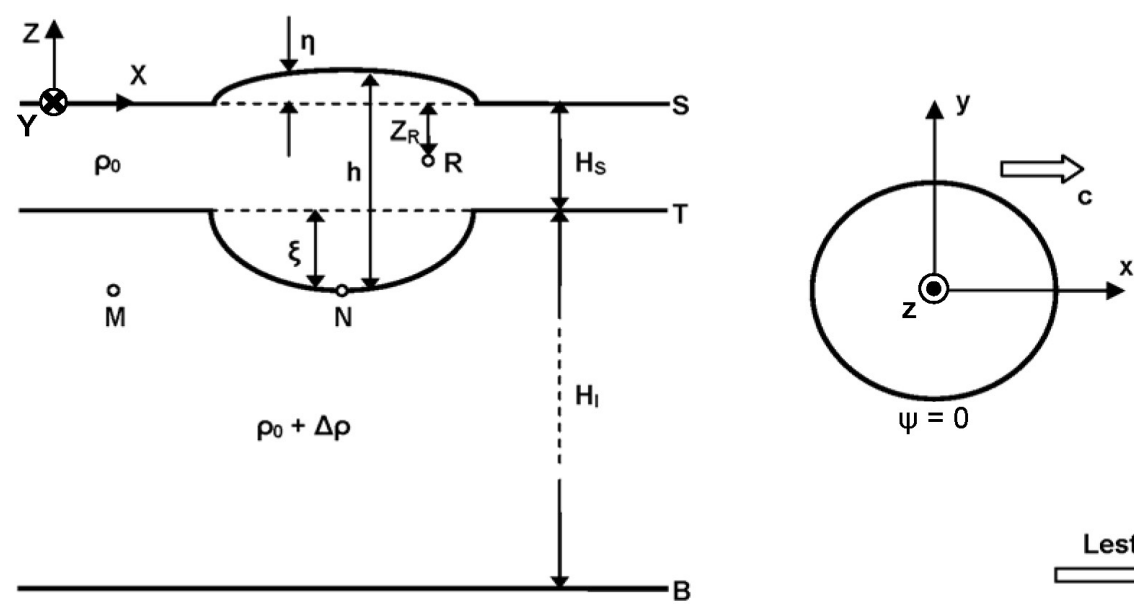

Figura 2 - Um vórtice anticiclônico se move para leste, com velocidade $c$, sobre uma camada abissal de um modelo de duas camadas. 0 esquema da esquerda mostra a estrutura vertical do modelo e suas principais variáveis assim como um sistema de coordenadas $X Y Z$ fixo na Terra. 0 esquema da direita mostra uma vista de topo do vórtice onde um sistema móvel de coordenadas $x y z$ está fixado no centro da feição. Não há proporção alguma entre as variáveis mostradas na figura.

$$
\begin{aligned}
& \frac{\partial V}{\partial T}+U \frac{\partial V}{\partial X}+V \frac{\partial V}{\partial Y}+\left(f_{0}+\beta Y\right) U \\
& =-\frac{1}{\rho_{0}} \frac{\partial P_{D}}{\partial Y} \\
& \frac{\partial U}{\partial X}+\frac{\partial V}{\partial Y}+\frac{\partial W}{\partial Z}=0, \\
& P_{D}=\rho_{0} g \eta .
\end{aligned}
$$

Nas Equações (1), (2) e (3) as variáveis $(U, V)$ são as componentes da velocidade horizontal nas direções zonal e meridional, respectivamente, as quais são independentes da profundidade, isto é, $\partial U / \partial Z=\partial V / \partial Z=0,0$ que acarreta $U=$ $U(X, Y, T)$ e $V=V(X, Y, T)$. A variável $W$ é a velocidade vertical; $P_{D}$ é a pressão dinâmica, onde $P_{D}=P_{D}(X, Y, T)$; $\rho_{0}$ é uma densidade de referência; $f_{0}$ é o parâmetro de Coriolis na latitude central $Y_{0}$ do plano- $\beta ; g$ é a aceleração da gravidade e $\beta$ é o fator de correção do parâmetro de Coriolis em função da latitude. A pressão total $P_{R}$ em um ponto qualquer $R$ (ver Fig. 2) contido no interior do vórtice isolado, de coordenada vertical $Z_{R}$, é dada por

$$
P_{R}=P_{a t m}+P_{D}-\rho_{0} g Z_{R}
$$

Na Equação (5), $P_{a t m}$ é a pressão atmosférica, a qual é suposta constante em qualquer ponto da superfície do mar. 0 terceiro termo do lado direito da Equação (5) é dependente da profundidade $Z_{R}$ do ponto $R$, enquanto o segundo termo é uma função de
( $X, Y, T)$. A utilização de (4) em (1) transforma esta expressão em

$$
\frac{\partial U}{\partial T}+U \frac{\partial U}{\partial X}+V \frac{\partial U}{\partial Y}-\left(f_{0}+\beta Y\right) V=-g \frac{\partial \eta}{\partial X} .
$$

0 fato da camada inferior ter sido assumida como estagnada implica na inexistência de gradientes de pressão nesta camada, 0 que faz com que as pressões nos pontos $\mathrm{M}$ e N (que estão numa mesma horizontal, ver Fig. 2) sejam iguais, desde que, por hipótese, o fluido está em equilíbrio hidrostático. As pressões nestes dois pontos são

$$
\begin{aligned}
& P_{M}=\rho_{0} g H_{S}+\left(\rho_{0}+\Delta \rho\right) g \xi \quad \mathrm{e} \\
& P_{N}=\rho_{0} g h=\rho_{0} g\left(\eta+H_{S}+\xi\right) .
\end{aligned}
$$

Fazendo-se $P_{M}=P_{N}$ com a utilização das expressões (7) conclui-se que

$$
\eta=\frac{\Delta \rho}{\rho_{0}} \xi
$$

A Equação (8) mostra uma relação muito importante em um modelo de duas camadas, em que a camada inferior é suposta abissal e estagnada, a qual determina que o deslocamento da superfície do mar $\eta$ é muito inferior que o respectivo deslocamento da interface $\xi$, o que faz com que a profundidade $h$ do vórtice isolado possa ser expressa por

$$
h=\eta+H_{S}+\xi \approx H_{S}+\xi .
$$

Utilizando-se a Equação (8) na expressão (6), esta transforma-se em

$$
\frac{\partial U}{\partial T}+U \frac{\partial U}{\partial X}+V \frac{\partial U}{\partial Y}-\left(f_{0}+\beta Y\right) V=-g \frac{\Delta \rho}{\rho_{0}} \frac{\partial \xi}{\partial X} .
$$


Introduzindo-se a gravidade reduzida $g^{\prime}=\left(\Delta \rho / \rho_{0}\right) g$ a Equação (10) resulta

$$
\frac{\partial U}{\partial T}+U \frac{\partial U}{\partial X}+V \frac{\partial U}{\partial Y}-\left(f_{0}+\beta Y\right) V=-g^{\prime} \frac{\partial \xi}{\partial X} .
$$

A Equação (9) permite escrever $\partial h / \partial x=\partial \xi / \partial x, 0$ que modifica a expressão (11) para

$$
\frac{\partial U}{\partial T}+U \frac{\partial U}{\partial X}+V \frac{\partial U}{\partial Y}-\left(f_{0}+\beta Y\right) V=-g^{\prime} \frac{\partial h}{\partial X} .
$$

As mesmas considerações, quando aplicadas na Equação (2), a transformam em

$$
\frac{\partial V}{\partial T}+U \frac{\partial V}{\partial X}+V \frac{\partial V}{\partial Y}+\left(f_{0}+\beta Y\right) U=-g^{\prime} \frac{\partial h}{\partial Y} .
$$

A integração vertical da Equação (3) da continuidade, ao longo da profundidade do vórtice isolado, tem como resultado (e.g., Cushman-Roisin, 1994)

$$
\left[\frac{\partial U}{\partial X}+\frac{\partial V}{\partial Y}\right] \int_{\eta-h}^{\eta} d Z+\left.W\right|_{\eta-h} ^{\eta}=0 .
$$

A equação da posição da superfície do oceano sobre o vórtice é dada por $z=\eta$, a qual derivada com relação ao tempo fornece

$$
\frac{d Z}{d T}=\left.W\right|_{\eta}=\frac{d \eta}{d T}=\frac{\partial \eta}{\partial T}+U \frac{\partial \eta}{\partial X}+V \frac{\partial \eta}{\partial Y} .
$$

Na obtenção da Equação (15) foi considerado que as partículas de fluido não podem deixar a superfície do mar. De mesma forma, a equação da posição vertical da termoclina pode ser dada por $z=\eta-h$, a qual derivada com relação ao tempo resulta em

$$
\begin{gathered}
\frac{d Z}{d T}=\left.\quad W\right|_{\eta-h}=\frac{d \eta}{d T}-\frac{d h}{d T}=\frac{\partial \eta}{\partial T}+U \frac{\partial \eta}{\partial X} \\
+V \frac{\partial \eta}{\partial Y}-\frac{\partial h}{\partial T}-U \frac{\partial h}{\partial X}-V \frac{\partial h}{\partial Y} .
\end{gathered}
$$

Na obtenção da Equação (16) foi assumido, de forma semelhante à Equação (15), que as partículas de fluido não podem atravessar o leito oceânico (fundo). A utilização das expressões (15) e (16) na Equação (14) a transforma em

$$
\left[\frac{\partial U}{\partial X}+\frac{\partial V}{\partial Y}\right] h+\frac{\partial h}{\partial T}+U \frac{\partial h}{\partial X}+V \frac{\partial h}{\partial Y}=0 .
$$

Reunindo-se alguns termos da expressão (17) obtém-se a forma final da equação da continuidade no modelo de águas rasas:

$$
\frac{\partial h}{\partial T}+\frac{\partial(h U)}{\partial X}+\frac{\partial(h V)}{\partial Y}=0 .
$$

As Equações (12), (13) e (18) são as equações de águas rasas escritas em um sistema de coordenadas fixo na Terra, as quais podem ser utilizadas para descrever os movimentos da camada superior do modelo de duas camadas mostrado na Figura 2.

Um novo sistema de coordenadas será agora instalado no centro do vórtice mostrado na Figura 2, o qual é assumido estar inicialmente transladando para leste com velocidade constante $c$. Neste sistema as variáveis independentes são $(x, y, z, t)$, que correspondem às coordenadas zonal, meridional e vertical e 0 tempo, respectivamente. Nos dois sistemas de coordenadas (fixo e móvel) os eixos $(X, x)$ e $(Y, y)$ são positivos para leste e para norte, respectivamente. É possível estabelecer as transformações de coordenadas $T=T(x, y, t), X=X(x, y, t)$ e $Y=Y(x, y, t)$ entre os dois sistemas. A relação entre as coordenadas verticais não é importante pois as componentes horizontais da velocidade $(u, v)$ são também independentes da profundidade. As expressões $x=X-c T, y=Y$ e $t=T$ podem ser estabelecidas, as quais relacionam algebricamente variáveis dos dois sistemas de coordenadas. A derivada temporal das duas primeiras expressões resulta

$$
\begin{aligned}
& \frac{\partial x}{\partial t}=\frac{\partial X}{\partial t}-c \frac{\partial T}{\partial t} \rightarrow u=U-c, \\
& \frac{\partial y}{\partial t}=\frac{\partial Y}{\partial t} \rightarrow v=V .
\end{aligned}
$$

Novos operadores derivativos podem ser desenvolvidos, os quais têm como expressões [onde, neste caso, $(\bullet)$ pode ser $U, V$ ou $h$ ]

$$
\begin{aligned}
\frac{\partial(\bullet)}{\partial T} & =\frac{\partial x}{\partial T} \frac{\partial(\bullet)}{\partial x}+\frac{\partial y}{\partial T} \frac{\partial(\bullet)}{\partial y}+\frac{\partial t}{\partial T} \frac{\partial(\bullet)}{\partial t} \\
& =-c \frac{\partial(\bullet)}{\partial x}+\frac{\partial(\bullet)}{\partial t}, \\
\frac{\partial(\bullet)}{\partial X} & =\frac{\partial x}{\partial X} \frac{\partial(\bullet)}{\partial x}+\frac{\partial y}{\partial X} \frac{\partial(\bullet)}{\partial y}+\frac{\partial t}{\partial X} \frac{\partial(\bullet)}{\partial t} \\
& =\frac{\partial(\bullet)}{\partial x}, \\
\frac{\partial(\bullet)}{\partial Y} & =\frac{\partial x}{\partial Y} \frac{\partial(\bullet)}{\partial x}+\frac{\partial y}{\partial Y} \frac{\partial(\bullet)}{\partial y}+\frac{\partial t}{\partial Y} \frac{\partial(\bullet)}{\partial t} \\
& =\frac{\partial(\bullet)}{\partial y} .
\end{aligned}
$$

Estes novos operadores serão aplicados nas equações de águas rasas (12), (13) e (18), visando escrevê-las no novo sistema móvel de coordenadas que se locomove de forma conjunta com 0 vórtice isolado. 0 movimento do vórtice neste novo sistema pode 
ser considerado estacionário caso seja assumido que a forma da feição não se altera muito ao longo do tempo (Nof, 1981a), ou seja, as variações temporais de $u, v, h$ e $\eta$ são nulas. Após a aplicação dos novos operadores as equações estacionárias de águas rasas, que serão utilizadas ao longo deste trabalho, têm como forma:

$$
\begin{aligned}
& u \frac{\partial u}{\partial x}+v \frac{\partial u}{\partial y}-\left(f_{0}+\beta y\right) v=-g^{\prime} \frac{\partial h}{\partial x}, \\
& u \frac{\partial v}{\partial x}+v \frac{\partial v}{\partial y}+\left(f_{0}+\beta y\right)(u+c)=-g^{\prime} \frac{\partial h}{\partial y}, \\
& \frac{\partial(u h)}{\partial x}+\frac{\partial(v h)}{\partial y}=0 .
\end{aligned}
$$

As equações transformadas (24), (25) e (26) descrevem 0 movimento de um vórtice isolado, sobre uma camada infinitamente profunda, em um sistema móvel de coordenadas que está estabelecido no centro da feição. Caso fossem supostos movimentos na camada inferior ocorreriam importantes interações entre 0 vórtice e esta camada (Nof, 1983) e o lado direito das Equações (24) e (25) seria modificado.

\subsection{A função de Bernoulli}

Nesta subseção a função de Bernoulli, descrita em um sistema móvel de coordenadas, será deduzida. Para tal, a Equação (24) deve ser inicialmente multiplicada por $u h$ e a Equação (25) por $v h$. A soma das duas expressões resultantes fornece

$$
\begin{aligned}
& u^{2} h \frac{\partial u}{\partial x}+v^{2} h \frac{\partial v}{\partial y}+u v h \frac{\partial u}{\partial y}+u v h \frac{\partial v}{\partial x} \\
& +\left(f_{0}+\beta y\right) c v h=-g^{\prime} u h \frac{\partial h}{\partial x}-g^{\prime} v h \frac{\partial h}{\partial y} .
\end{aligned}
$$

A partir da Equação (26), as seguintes expressões podem ser estabelecidas (e.g., Fox \& McDonald, 1978):

$$
\frac{\partial \psi}{\partial x}=v h \quad \frac{\partial \psi}{\partial y}=-u h .
$$

Na expressão (28), $\psi=\psi(x, y)$ é uma função de transporte (pois cada uma das expressões está multiplicada pela profundidade $h$ ), a qual é constante ao longo de uma linha de corrente. Curvas instantâneas que tangenciam os vetores velocidade de um escoamento são chamadas de linhas de corrente (e.g., Kundu \& Cohen, 2008). A existência desta função é uma consequência da continuidade da massa e da incompressibilidade do fluido (e.g., Milne-Thomson, 1968). A utilização das expressões (28) na Equação (27) resulta

$$
\begin{gathered}
\frac{\partial \psi}{\partial x}\left[u \frac{\partial u}{\partial y}+v \frac{\partial v}{\partial y}\right]-\frac{\partial \psi}{\partial y}\left[u \frac{\partial u}{\partial x}+v \frac{\partial v}{\partial x}\right] \\
+\left(f_{0}+\beta y\right) c \frac{\partial \psi}{\partial x}-\frac{\partial \psi}{\partial y} \frac{\partial\left(g^{\prime} h\right)}{\partial x} \\
+\frac{\partial \psi}{\partial x} \frac{\partial\left(g^{\prime} h\right)}{\partial y}=0 .
\end{gathered}
$$

A expressão (29) pode ainda ser reescrita na forma

$$
\begin{gathered}
\frac{\partial \psi}{\partial x}\left[\frac{1}{2} \frac{\partial u^{2}}{\partial y}+\frac{1}{2} \frac{\partial v^{2}}{\partial y}\right]-\frac{\partial \psi}{\partial y}\left[\frac{1}{2} \frac{\partial u^{2}}{\partial x}+\frac{1}{2} \frac{\partial v^{2}}{\partial x}\right] \\
+\frac{\partial \psi}{\partial x} \frac{\partial}{\partial y}\left[\left(f_{0} y+\beta y^{2} / 2\right) c\right]-\frac{\partial \psi}{\partial y} \frac{\partial\left(g^{\prime} h\right)}{\partial x} \\
+\frac{\partial \psi}{\partial x} \frac{\partial\left(g^{\prime} h\right)}{\partial y}=0 .
\end{gathered}
$$

onde a reunião de termos semelhantes transforma a Equação (30) em

$$
\begin{gathered}
\frac{\partial \psi}{\partial x} \frac{\partial}{\partial y}\left[\frac{u^{2}+v^{2}}{2}+g^{\prime} h+\left(f_{0}+\beta y / 2\right) c y\right] \\
-\frac{\partial \psi}{\partial y} \frac{\partial}{\partial x}\left[\frac{u^{2}+v^{2}}{2}+g^{\prime} h+\left(f_{0}+\beta y / 2\right) c y\right]=0 .
\end{gathered}
$$

Esta Equação (31) pode ser escrita na forma de um Jacobiano, 0 que fornece

$$
J\left[\psi, \frac{u^{2}+v^{2}}{2}+g^{\prime} h+\left(f_{0}+\beta y / 2\right) c y\right]=0 .
$$

A expressão da direita dentro dos colchetes define a função de Bernoulli $B$ (neste caso, escrita para um sistema móvel de coordenadas com velocidade zonal $c$ ) que é dada por

$$
B=\frac{u^{2}+v^{2}}{2}+g^{\prime} h+\left(f_{0}+\beta y / 2\right) c y .
$$

A consideração das propriedades de Jacobianos permite concluir, a partir da Equação (32), que a função de Bernoulli $B$ é constante ao longo de uma linha de corrente, ou seja

$$
\frac{u^{2}+v^{2}}{2}+g^{\prime} h+\left(f_{0}+\beta y / 2\right) c y=K(\psi) .
$$

Na Equação (34), $K(\psi)$ é uma constante que se modifica de uma linha de corrente para outra. A função de Bernoulli é composta por termos associados com a energia cinética do sistema e 
com a sua energia potencial [primeiro e segundo termos do lado esquerdo da expressão (34), respectivamente]. 0 último termo deste mesmo lado esquerdo decorre do fato do sistema de coordenadas utilizado estar em movimento. Este termo seria nulo em um sistema fixo de coordenadas, quando então $c=0$.

\subsection{A equação integrada do momentum meridional}

Nesta subseção a equação meridional do momentum, (25), será integrada sobre toda a superfície do vórtice. Este procedimento visa a obtenção de uma expressão para a velocidade de translação $c$ desta feição (inicialmente suposta para leste). A expressão (25), multiplicada pela profundidade $h$, resulta

$$
u h \frac{\partial v}{\partial x}+v h \frac{\partial v}{\partial y}+\left(f_{0}+\beta y\right)(u h+c h)=-g^{\prime} h \frac{\partial h}{\partial y} .
$$

Esta expressão (35) pode ser reescrita na forma

$$
\begin{gathered}
\frac{\partial(u v h)}{\partial x}-v \frac{\partial(u h)}{\partial x}+\frac{\partial\left(h v^{2}\right)}{\partial y}-v \frac{\partial(v h)}{\partial y} \\
+\left(f_{0}+\beta y\right)(u h+c h)=-\frac{g^{\prime}}{2} \frac{\partial h^{2}}{\partial y} .
\end{gathered}
$$

A soma dos segundo e quarto termos do lado esquerdo da Equação (36) resulta nula tendo em vista a equação estacionária da continuidade (26). Com esta consideração a expressão (36) resulta

$$
\begin{gathered}
\frac{\partial(u v h)}{\partial x}+\frac{\partial\left(h v^{2}\right)}{\partial y}+\left(f_{0}+\beta y\right) u h \\
+\left(f_{0}+\beta y\right) c h=-\frac{g^{\prime}}{2} \frac{\partial h^{2}}{\partial y} .
\end{gathered}
$$

A integração da Equação (37) sobre toda a área horizontal $A$ do vórtice isolado fornece como resultado

$$
\begin{gathered}
\iint_{A} \frac{\partial(u v h)}{\partial x} d x d y+\iint_{A} \frac{\partial\left(h v^{2}\right)}{\partial y} d x d y \\
+\iint_{A}\left(f_{0}+\beta y\right) u h d x d y \\
+\iint_{A}\left(f_{0}+\beta y\right) c h d x d y=-\iint_{A} \frac{g^{\prime}}{2} \frac{\partial h^{2}}{\partial y} d x d y .
\end{gathered}
$$

A utilização das expressões (28) na Equação (38) transforma esta em

$$
\begin{gathered}
\iint_{A} \frac{\partial(u v h)}{\partial x} d x d y+\iint_{A} \frac{\partial\left(h v^{2}\right)}{\partial y} d x d y \\
-f_{0} \iint_{A} \frac{\partial \psi}{\partial y} d x d y-\beta \iint_{A}\left[\frac{\partial(y \psi)}{\partial y}-\psi\right] d x d y \\
+c \iint_{A}\left(f_{0}+\beta y\right) h d x d y=-\frac{g^{\prime}}{2} \iint_{A} \frac{\partial h^{2}}{\partial y} d x d y .
\end{gathered}
$$

0 Teorema de Stokes estabelece que uma integral de superfície pode ser transformada em uma integral de linha de acordo com:

$$
\iint_{A}(\partial m / \partial x-\partial n / \partial y) d x d y=\oint_{\phi}[n d x+m d y],
$$

onde $m=m(x, y)$ e $n=n(x, y)$.

A seta da integral de linha do lado direito da expressão (40) indica que esta integral é realizada no sentido anti-horário ao longo do contorno $\phi$, sendo a normal à superfície de integração $A$ definida na direção positiva de $z$. A aplicação do Teorema de Stokes na expressão (39) a transforma em

$$
\begin{gathered}
\oint_{\phi} v h[u d y-v d x]+f_{0} \oint_{\phi} \psi d x+\beta \oint_{\phi} y \psi d x \\
+\beta \iint_{A} \psi d x d y+c \iint_{A}\left(f_{0}+\beta y\right) h d x d y \\
=\frac{g^{\prime}}{2} \oint_{\phi} h^{2} d x .
\end{gathered}
$$

A Figura 3 mostra duas linhas de corrente de um determinado escoamento, de profundidade $h$, as quais são separadas por distâncias infinitesimais.

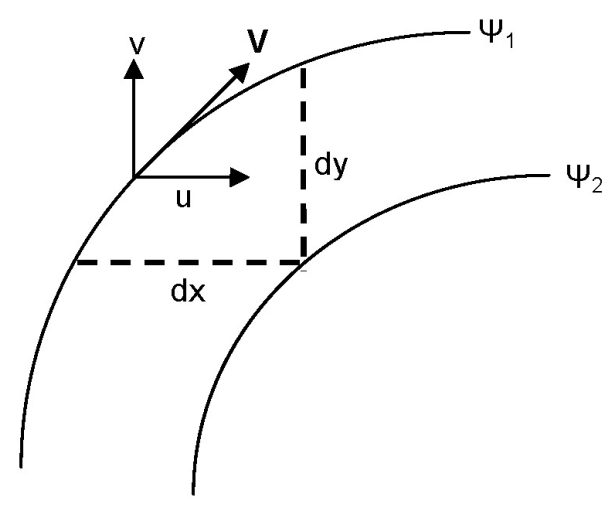

Figura 3 - Linhas de corrente de um determinado escoamento.

Por definição, a diferença entre as funções (corrente) transporte de duas linhas de corrente fornece 0 transporte de volume 
entre estas duas linhas (e.g., Milne-Thomson, 1968; Batchelor, 1998). Além disso, o transporte de volume que atravessa uma linha de corrente é nulo. Com estas considerações, o transporte que atravessa a linha tracejada horizontal da Figura 3 , dado por $v h d x$, deve equivaler ao transporte que atravessa a linha tracejada vertical da mesma figura, o qual é dado por $u h d y$. Esta igualdade entre os dois transportes permite definir a relação $v d x=u d y$, a qual mostra que a primeira integral do lado esquerdo da Equação (41) é nula. A conveniente escolha de $\psi=0$ na linha de corrente livre que circula 0 vórtice isolado (ver 0 esquema da direita na Fig. 2) torna nulas as segunda e terceira integrais de linha da expressão (41). A expressão final da equação integrada do momentum meridional passa então a ser (conforme Nof, 1983)

$$
\begin{gathered}
\beta \iint_{A} \psi d x d y+c \iint_{A}\left(f_{0}+\beta y\right) h d x d y \\
=\frac{g^{\prime}}{2} \oint_{\phi} h^{2} d x .
\end{gathered}
$$

\section{AS FORÇAS MERIDIONAIS}

Esta seção apresenta as forças meridionais que atuam sobre vórtices isolados, em movimento de translação. A Equação (42) sinaliza para a existência de três destas forças, as quais são: (a) a força devida ao efeito $\beta$ no interior do vórtice isolado, (b) a força de Coriolis que atua no sentido de defletir o movimento de translação da feição para a esquerda (direita) no hemisfério Sul (Norte) e (c) a força devida à atuação do fluido circunvizinho ao vórtice sobre a feição, a qual é chamada de força ambiente. Estas três forças correspondem aos três termos da Equação (42), da esquerda para a direita, respectivamente. A partir deste ponto estas forças serão discutidas isoladamente e seus respectivos termos serão analisados e, quando necessário, reapresentados matematicamente. Cabe destacar que os termos da Equação (42) não possuem uma dimensão explícita de força. Contudo, tendose em vista que nesta análise o centro de massa do vórtice isolado não se desloca meridionalmente, ou seja, a quantidade de movimento meridional da feição é nula, pode-se afirmar que a resultante das forças externas meridionais que agem sobre 0 vórtice isolado é nula. Esta consideração faz com que a Equação (42) possa ser então tratada como um balanço de forças meridionais.

\subsection{A força beta $(\beta)$}

Todas as partículas de fluido que descrevem um movimento rotativo ao redor do centro do vórtice isolado estão sujeitas à ação da força de Coriolis que tende a defletir o movimento de cada uma delas para o lado esquerdo (direito) no hemisfério Sul (Norte). A Figura 4 mostra duas partículas de fluido (representadas pelos quadrados pretos) que passam pelo hemisfério Norte de um vórtice isolado anticiclônico que translada zonalmente no hemisfério Sul do planeta. As partículas possuem velocidade orbital $V_{\theta}$ e sofrem ação da força de Coriolis $(F C)$ conforme mostram as setas cinzas da Figura 4. Estas forças $F C$ podem ser decompostas em duas componentes, sendo uma zonal (setas hachuradas na figura) e outra meridional (setas brancas menores na figura). Como se pode verificar na Figura 4, as componentes zonais da FC sempre se anularão entre si enquanto as componentes meridionais se somam, criando-se então uma força líquida $\left(F C_{L}\right)$ que, no caso do hemisfério Norte do vórtice isolado, aponta para sul (representada pela seta branca mais larga na figura). A mesma situação ocorre no hemisfério Sul da feição resultando então uma $F C_{L}$ que agora aponta para norte

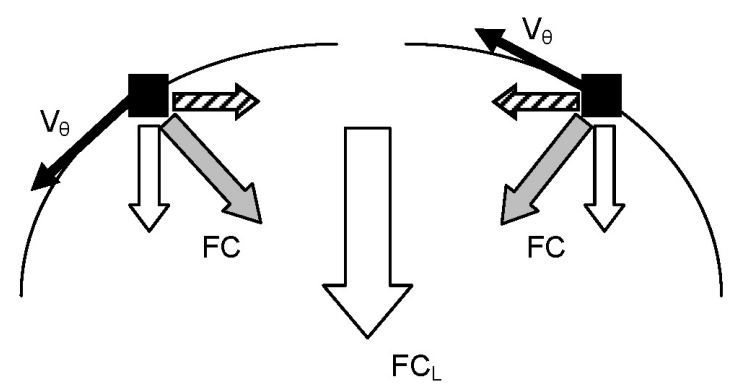

Figura 4 - Forças que atuam nas partículas de fluido que passam pelo hemisfério Norte de um vórtice anticiclônico que translada zonalmente pelo hemisfério Sul da Terra. 0 vetor preto representa a velocidade $v_{\theta}$ destas partículas, a seta cinza é a força de Coriolis, as setas branca (pequena) e hachurada são as componentes meridional e zonal desta força, respectivamente, e a seta branca grande representa a força de Coriolis resultante sobre a feição devido aos movimentos representados.

Como 0 parâmetro de Coriolis é mais intenso próximo aos pólos devido à ação de $\beta$, a $F C_{L}$ do hemisfério Sul do vórtice isolado é mais intensa (para o caso de um vórtice no hemisfério Sul geográfico) que a sua análoga do hemisfério Norte da feição. Isto resulta numa força interna do vórtice, devida ao efeito $\beta$, que aponta para a linha do Equador, no caso de vórtices isolados anticiclônicos, e para os pólos, no caso de feições ciclônicas. Voltando-se ao primeiro termo do lado esquerdo da Equação (42), 0 qual corresponde à força $\beta$ interna ao vórtice isolado, podese constatar que esta força será nula na ausência do parâmetro $\beta$ (para um estudo realizado no plano- $f$, por exemplo), tendo em vista que 0 termo é uma função direta de $\beta$. Outra interessante constatação é que a inversão do sentido da força $\beta$ entre vórtices isolados ciclônicos e anticiclônicos é mostrada no termo matemático correspondente. A função corrente média $\psi$ 
contida neste termo possui sinal contrário de um tipo de vórtice para outro, sendo negativa (positiva) para vórtices anticiclônicos (ciclônicos) no hemisfério Sul do planeta. Esta troca de sinais é melhor discutida na última seção deste artigo.

\subsection{A força de Coriolis}

0 segundo termo do lado esquerdo da Equação (42) corresponde à força de Coriolis, que tende a defletir o movimento de translação do vórtice isolado para a esquerda (direita) no hemisfério Sul (Norte) do planeta. Este termo pode ser reescrito numa forma mais conveniente para uma análise de escala, o que é mostrado no lado esquerdo da expressão (43):

$$
c f_{0} \iint_{A}\left(1+\beta y / f_{0}\right) h d x d y \approx c f_{0} \iint_{A} h d x d y .
$$

Utilizando-se como parâmetros típicos para 0 vórtice isolado $\beta=2.10^{-11} \mathrm{~m}^{-1} \mathrm{~s}^{-1}, L=100 \mathrm{~km}$ (ou $10^{5} \mathrm{~m}$ ) e $f_{0}=$ $10^{-4} s^{-1}$, onde $L$ é a sua escala típica de comprimento, 0 termo $\beta y / f_{0}$ resulta $2.10^{-2}$ e pode ser negligenciado, 0 que justifica 0 termo do lado direito da Equação (43), o qual permitirá 0 cálculo da força de Coriolis que atua sobre 0 vórtice isolado tendo-se a velocidade de translação $c$ da feição. Cabe destacar que a integral do lado direito de (43) corresponde ao volume do vórtice isolado.

\subsection{A força ambiente}

0 termo do lado direito da Equação (42) é, sem dúvida alguma, 0 de mais difícil interpretação e aquele que exige um maior tratamento matemático para ser melhor visualizado. A Figura 5 mostra 0 vórtice isolado, com movimento translacional para leste com velocidade zonal $c$, onde uma linha de corrente $\mathrm{ABCD}$ com função de transporte $\psi$ é destacada. Esta linha procede de um ponto $\mathrm{A}$ muito à frente do vórtice e encontra a feição no ponto $\mathrm{B}$ onde a velocidade do fluido externo é trazida até o repouso. Este ponto é chamado ponto de estagnação (e.g., Milne-Thomson, 1968). 0 contorno do vórtice fará parte desta linha de corrente, a qual se reúne novamente no ponto $C$ da periferia do vórtice, um segundo ponto de estagnação, procedendo daí até 0 ponto D situado a uma grande distância da feição. Linhas de corrente com esta configuração são conhecidas como linhas de corrente divisórias (e.g., Milne-Thomson, 1968). Na verdade, os pontos A, B, C e D não precisam estar no mesmo alinhamento, conforme mostra a Figura 5. 0 ponto A (origem da linha de corrente considerada) pode ter um desvio meridional com relação à reta que passa pelos pontos $\mathrm{B}$ e $\mathrm{C}$, ou seja, $y_{A} \neq 0,0$ mesmo ocorrendo com o ponto $\mathrm{D}$ (este último desvio não é mostrado na figura).
Tendo-se em vista o sistema móvel de coordenadas instalado no centro do vórtice isolado, que se move com velocidade zonal $c$, 0 fluido externo deslocado pela feição (contido na camada superior da Figura 2, onde a interface e a camada superior do oceano são planas) se move na direção do vórtice com velocidade $-c$ e profundidade $H_{S}$. Doravante esta profundidade será tratada apenas como $H$, a qual corresponde à profundidade da camada superior externa ao vórtice, a qual é nula no caso de vórtices do tipo lente (feições em que a termoclina do vórtice encontra a superfície do oceano nas suas bordas). A aplicação da função de Bernoulli, definida na seção anterior pela Equação (34), ao longo da linha de corrente mostrada na Figura 5 permite escrever

$$
\frac{u^{2}+v^{2}}{2}+g^{\prime} h+\left(f_{0}+\beta y / 2\right) c y=\frac{c^{2}}{2}+g^{\prime} H .
$$

0 lado direito da expressão (44) corresponde à constante $K(\psi)$ da Equação (34) e descreve 0 valor da função de Bernoulli em um ponto situado muito à frente do vórtice isolado (ponto $\mathrm{A}$, ver Fig. 5). 0 termo associado ao parâmetro de Coriolis não consta do lado direito da Equação (44) em virtude de ter sido considerado que a posição meridional $y_{A}$ do ponto $\mathrm{A}$ é muito inferior à unidade (Nof, 1983), ou de ordem $\epsilon R_{D}$, onde $\epsilon=$ $\beta r_{0} / f_{0}$ ( $r_{0}$ é o raio do vórtice) é um parâmetro adimensional que mede a variação do parâmetro de Coriolis ao longo de um dos hemisférios do vórtice isolado e $R_{D}$ é o raio de deformação desta feição. De fato, para valores típicos tais como $r_{0}=100$ $\mathrm{km}, R_{D}=30 \mathrm{~km}, c=10^{-2} \mathrm{~m} \cdot \mathrm{s}^{-1}, f_{0}=10^{-4} \mathrm{~s}^{-1}$ e $\beta=$ $2.10^{-11} \mathrm{~m}^{-1} \mathrm{~s}^{-1}$ o parâmetro $\epsilon$ resulta $2.10^{-2}$ e 0 termo $f_{0} c y$ relativo ao ponto $A$ pode ser negligenciado.

A análise de escala realizada na subseção anterior mostra que 0 termo $\beta y / f_{0}$ é negligenciável. Assumindo-se que a ordem das velocidades horizontais é a mesma de $c, 0$ termo $\left(u^{2}+\right.$ $\left.v^{2}\right) / f_{0} c y$ transforma-se em $c / f_{0} y, 0$ qual possui ordem $10^{-3}$ (utilizando-se os valores típicos citados anteriormente) e também pode ser negligenciado. Com estas considerações de escala a Equação (44) pode ser reescrita na forma

$$
g^{\prime} h+f_{0} c y=c^{2} / 2+g^{\prime} H .
$$

Esta equação será temporariamente deixada de lado. 0 foco agora passa a ser a relação entre o movimento do vórtice isolado e do fluido externo ao seu redor. À medida que 0 vórtice translada ele carrega toda a sua anomalia de massa, 0 que faz com que a camada superior de fluido tenha que ser deslocada, à frente da feição, para compensar o movimento de todo o volume do vórtice. Conforme Nof (1983), o comportamento do campo externo nesta situação é similar à reação deste à passagem de 


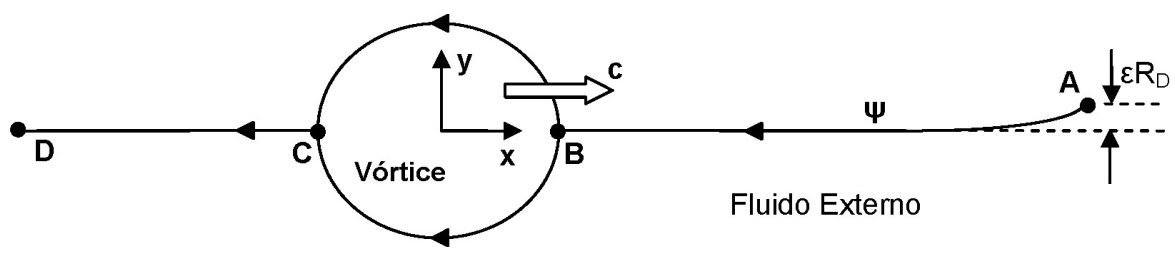

Figura 5 - Linha de corrente divisória em um vórtice isolado que possui movimento de translação para leste, com velocidade $c$. A função de Bernoulli descrita no texto será aplicada nesta linha de corrente. Não há proporção alguma na figura.

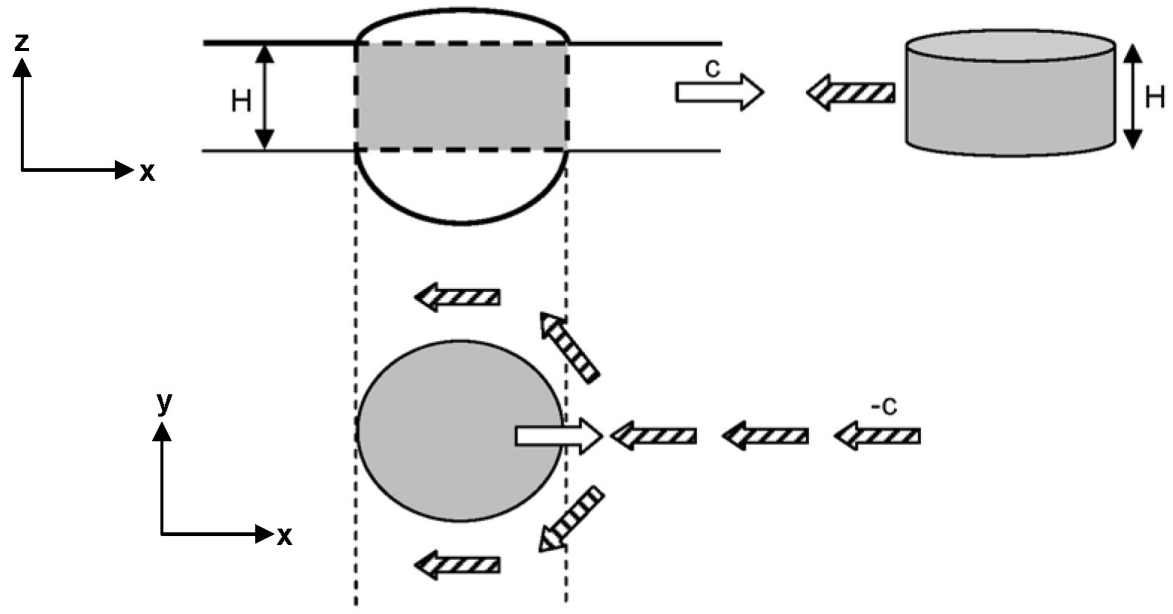

Figura 6 - Vórtice isolado anticiclônico em movimento zonal para leste. A área cinza do esquema superior esquerdo mostra a seção lateral do volume de fluido externo que será deslocado pelo vórtice, o qual corresponde ao volume de um cilindro (esquema superior à direita) com mesma seção superficial do vórtice e altura $H$ da camada superior. 0 esquema inferior mostra a distribuição do fluido externo deslocado (indicado pelas setas hachuradas) pelo vórtice nos lados norte e sul da feição. Este fluido se desloca para oeste com relação ao sistema de coordenadas $x y z$ do vórtice. Não há proporção alguma entre as dimensões do vórtice mostradas na figura.

um cilindro sólido através da camada superior. Utilizando-se novamente como exemplo um vórtice anticiclônico, a Figura 6 mostra que 0 volume de fluido externo deslocado pela feição, em seu movimento zonal para leste, corresponde ao volume de um cilindro de mesma seção horizontal do vórtice e altura $H$.

0 transporte do fluido externo é distribuído ao longo de duas regiões circunvizinhas ao vórtice isolado (ver Fig. 6), situadas ao norte e ao sul da feição. A largura destas regiões deve ser, no mínimo, da ordem do raio de Rossby do vórtice (Nof, 1983). Como o movimento de vórtices isolados devido à autopropulsão é muito lento (da ordem de $1 \mathrm{~cm} . \mathrm{s}^{-1}$ ou aproximadamente $1 \mathrm{~km} / \mathrm{dia}$ ), os fluxos do fluido externo, laterais ao vórtice, podem ser assumidos como geostróficos. A Figura 7 mostra uma visão lateral do vórtice isolado do ponto de vista de um observador instalado à oeste e olhando direto para leste, no hemisfério Sul. No desenvolvimento inicial deste estudo foi suposto que 0 vórtice se movimenta para leste com velocidade $c, 0$ que justifica 0 vórtice estar se afastando do observador.
A presença dos fluxos geostróficos devido ao movimento do fluido externo e a necessidade de continuidade da superfície do mar e da termoclina fazem com que o perfil do vórtice tenha que se adequar ao campo externo, conforme mostra a Figura 7. A seta branca indica a força devida ao gradiente de pressão que age sobre as partículas do fluido externo em movimento e a seta cinza mostra a força de Coriolis que atua sobre estas mesmas partículas (na Fig. 7 estas forças só estão sendo mostradas no braço norte do fluido externo em movimento). Estas forças estão em balanço geostrófico.

A força norte-sul $F$ (por unidade de profundidade) que age sobre o vórtice isolado pode ser calculada por

$$
F=\oint_{\phi} P d x=\rho g \oint_{\phi} h d x .
$$

Isolando-se o termo $h$ na Equação (45) e substituindo-se em (46) obtém-se

$F=\oint_{\phi} P d x=\rho\left(g / g^{\prime}\right) \oint_{\phi}\left(c^{2} / 2+g^{\prime} H-f_{0} c y\right) d x$. 


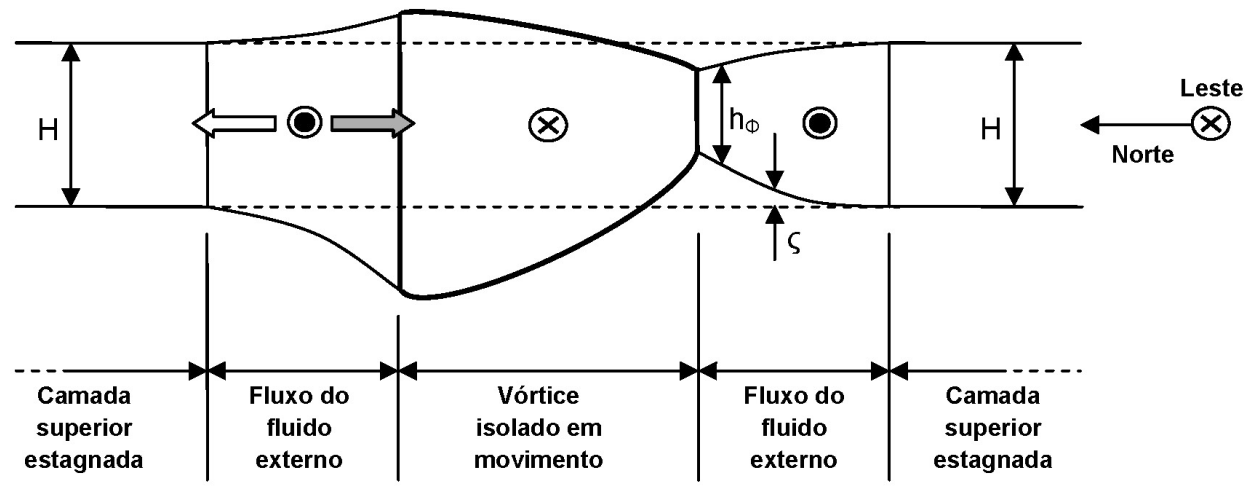

Figura 7 - Perfil lateral de um vórtice isolado, em movimento zonal inicialmente suposto para leste (ver texto), sofrendo a ação do campo externo circunvizinho. A deformação da feição se deve a sua acomodação ao campo de pressão gerado pelo movimento do fluido externo à volta do vórtice. A seta branca da esquerda indica a força do gradiente de pressão e a seta cinza indica a força de Coriolis. Estas forças, apesar de presentes, não são mostradas no lado externo sul da feição. Não há proporção alguma entre as dimensões e variáveis mostradas na figura.

Como os dois primeiros termos da integral de linha são constantes, a expressão (47) transforma-se em

$$
F=-f_{0} c \rho\left(g / g^{\prime}\right) \oint_{\phi} y d x
$$

E a força norte-sul total $F_{T}$ que age sobre 0 vórtice, com a utilização da expressão (48), tem como expressão

$$
\begin{gathered}
F_{T}=\int_{-h_{\phi}}^{0} F d z=F \int_{-h_{\phi}}^{0} d z \\
=-f_{0} c \rho\left(g / g^{\prime}\right) H \oint_{\phi} y d x .
\end{gathered}
$$

Na expressão (49) $h_{\phi}$ é a profundidade na borda do vórtice isolado, expressa por $h_{\phi}=H+\zeta \approx H$, onde $\zeta$ é o deslocamento da termoclina na região de movimento do fluido externo (o deslocamento da superfície do mar foi negligenciado na expressão de $\left.h_{\phi}\right)$. Com esta consideração, o terceiro termo da Equação (42) pode ser reescrito na seguinte forma

$$
\begin{gathered}
\frac{g^{\prime}}{2} \oint_{\phi} h^{2} d x=\frac{g^{\prime}}{2} \oint_{\phi}(H+\zeta)^{2} d x \\
=\frac{g^{\prime}}{2} \oint_{\phi}\left(H^{2}+2 H \zeta+\zeta^{2}\right) d x \approx g^{\prime} H \oint_{\phi} \zeta d x,
\end{gathered}
$$

desde que $H$ é constante e $\zeta \ll H$.

A força norte-sul total $F_{T}$ pode ainda ser expressa de uma outra maneira. Partindo-se novamente da expressão (46) pode- se escrever

$$
\begin{gathered}
F=\oint_{\phi} P d x=\rho g \oint_{\phi} h d x \\
=\rho g \oint_{\phi}(H+\zeta) d x=\rho g \oint_{\phi} \zeta d x .
\end{gathered}
$$

A expressão (51) conduz a uma nova expressão para $F_{T}$ que é dada por:

$$
\begin{gathered}
F_{T}=\int_{-h_{\phi}}^{0} F d z=F \int_{-h_{\phi}}^{0} d z \\
=\rho g(H+\zeta) \oint_{\phi} \zeta d x \approx \rho g H \oint_{\phi} \zeta d x .
\end{gathered}
$$

A comparação das Equações (50) e (52) permite escrever a relação

$$
\frac{g^{\prime}}{2} \oint_{\phi} h^{2} d x=\frac{F_{T}}{\rho\left(g / g^{\prime}\right)} .
$$

Substituindo-se a Equação (49) em (53) obtém-se então

$$
\frac{g^{\prime}}{2} \oint_{\phi} h^{2} d x=-f_{0} c H \oint_{\phi} y d x .
$$

A aplicação do Teorema de Stokes em (54) permite a obtenção da expressão final da força ambiente que age sobre 0 vórtice isolado em movimento, que é dada por

$$
\begin{gathered}
\frac{g^{\prime}}{2} \oint_{\phi} h^{2} d x=f_{0} c H \iint_{A} \frac{\partial y}{\partial y} d y d x \\
=f_{0} c \iint_{A} H d y d x .
\end{gathered}
$$


Na expressão (55) a integral descreve o volume de fluido externo deslocado com a passagem do vórtice isolado. Esta expressão é similar à Equação (43) que descreve a força de Coriolis que atua sobre o movimento de translação do vórtice isolado. Cabe destacar que naquelas situações em que não há atuação do fluido externo sobre o vórtice isolado em movimento (como, por exemplo, no caso de vórtices do tipo lente), o termo $\left(g^{\prime} / 2\right) \oint_{\phi} h^{2} d x$ resultará nulo de acordo com a expressão (55), pois $H=0$, comprovando a inexistência de uma força ambiente.

\section{A DERIVA PARA OESTE}

Nesta seção, a expressão final que permite o cálculo da velocidade $c$ de translação de vórtices isolados será apresentada. A equação integrada do momentum meridional (42) pode ser reescrita com a utilização das expressões (43) e (55), o que resulta:

$$
\begin{gathered}
\beta \iint_{A} \psi d x d y+c f_{0} \iint_{A} h d x d y \\
=c f_{0} \iint_{A} H d x d y .
\end{gathered}
$$

A Equação (56) permite a obtenção da expressão que fornece a velocidade aproximada $c$ de translação de vórtices isolados, a qual é dada por

$$
c=-\frac{\beta \iint_{A} \psi d x d y}{f_{0} \iint_{A}(h-H) d x d y} .
$$

A integral no denominador da expressão (57) corresponde à diferença entre os volumes do vórtice isolado e 0 de fluido externo deslocado pelo movimento do vórtice. Uma análise no sinal desta velocidade $c$ será agora realizada e, para tal, serão analisados vórtices isolados ciclônicos e anticiclônicos em movimento no hemisfério Sul do planeta.

(a) Vórtice anticiclônico com formato lente: para este tipo de vórtice tem-se $H=0$ e nenhum volume de fluido externo é deslocado, não havendo então força ambiente atuando sobre 0 vórtice em movimento. Como a função corrente média $\psi$ é negativa no interior de vórtices anticiclônicos no hemistério Sul, assim como o parâmetro de Coriolis $f_{0}$, a expressão (57) resulta:

$$
c=-\frac{\beta \iint_{A} \psi d x d y}{f_{0} \iint_{A} h d x d y}=-\frac{(-)}{(-)}<0 .
$$

A expressão (58) mostra que a velocidade $c$ é negativa, ou seja, este tipo de vórtice translada, de forma autopropulsionada, no sentido oeste. A Figura 8 mostra este vórtice anticiclônico, que é caracterizado por sua termoclina aflorar na superfície do mar nas suas bordas. Em termos de um modelo de duas camadas a camada superior corresponde exatamente ao líquido contido no interior do vórtice.

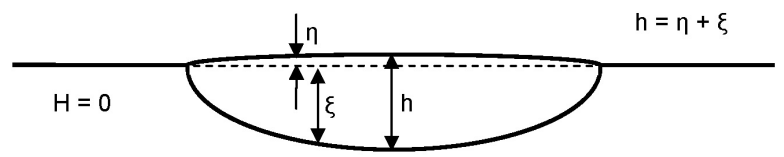

Figura 8 - Vórtice anticiclônico com formato lente. Este tipo de vórtice gira em sentido anti-horário no hemisfério Sul e a profundidade da camada superior externa ao vórtice é nula. Não há proporção alguma entre as variáveis mostradas na figura.

(b) Vórtice anticiclônico com formato não-lente: neste tipo de vórtice tem-se $h>H$ e a expressão (57) agora resulta:

$$
c=-\frac{\beta \iint_{A} \psi d x d y}{f_{0} \iint_{A}(h-H) d x d y}=-\frac{(-)}{(-)}<0 .
$$

A expressão (59) mostra que a velocidade $c$ ainda é negativa, ou seja, este tipo de vórtice também translada, de forma autopropulsionada, no sentido oeste. A Figura 9 mostra este vórtice, 0 qual difere do anterior devido ao fato de que a profundidade da camada superior externa ao vórtice é agora não nula, ou seja, haverá deslocamento de fluido externo devido à translação do vórtice isolado.

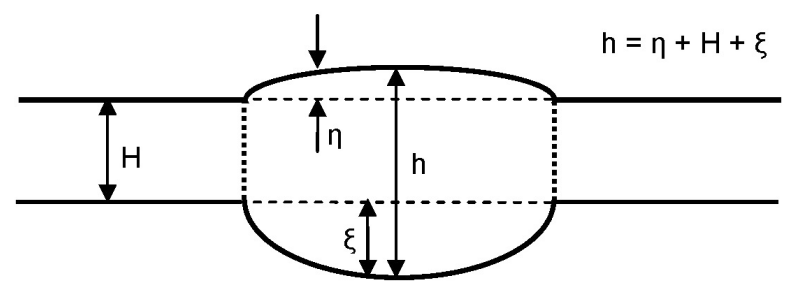

Figura 9 - Vórtice anticiclônico com formato não-lente. Este tipo de vórtice gira em sentido anti-horário no hemisfério Sul e a profundidade da camada superior externa ao vórtice não é nula. Não há proporção alguma entre as variáveis mostradas na figura.

(c) Vórtice ciclônico com formato não-lente: neste tipo de vórtice tem-se $h<H$. A função corrente média $\psi$ é positiva no interior de vórtices ciclônicos no hemisfério Sul. Com estas considerações a expressão (57) agora resulta:

$$
c=-\frac{\beta \iint_{A} \psi d x d y}{f_{0} \iint_{A}(h-H) d x d y}=-\frac{(+)}{(+)}<0 .
$$

A expressão (60) mostra que a velocidade $c$ ainda é negativa, ou seja, os vórtices ciclônicos também transladam, de forma autopropulsionada, no sentido oeste. A Figura 10 mostra o perfil de um vórtice ciclônico. 


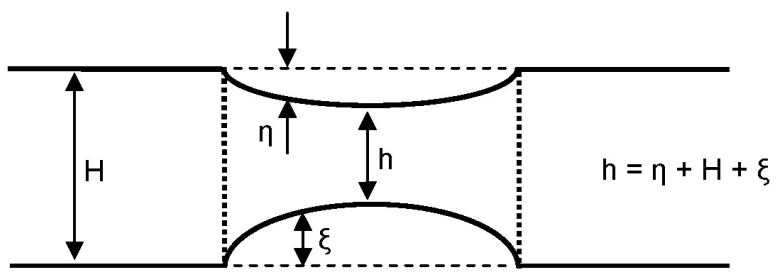

Figura 10 - Vórtice ciclônico com formato não-lente. Este tipo de vórtice gira em sentido horário no hemisfério Sul e a profundidade da camada superior externa ao vórtice não é nula. Não há proporção alguma entre as variáveis mostradas na figura.

Como se verifica nesta seção, todo o vórtice isolado no hemisfério Sul geográfico, no plano- $\beta$, é autopropulsionado para oeste. A conclusão é a mesma se a análise for estendida para 0 hemisfério Norte geográfico.

\section{O BALANÇO MERIDIONAL DE FORÇAS}

Nesta seção as forças que atuam meridionalmente sobre vórtices isolados, em movimento no hemisfério Sul, serão analisadas e comparadas. Conforme mostra a Equação (42), reescrita na forma da expressão (56), três forças competem meridionalmente durante a translação do vórtice isolado. Esta última expressão é aqui reproduzida, e cada termo é devidamente nomeado, conforme a força a ele associada.

$$
\underbrace{\beta \iint_{A} \psi d x d y}_{\text {Força Beta }}+\underbrace{c f_{0} \iint_{A} h d x d y}_{\text {Força Coriolis }}-\underbrace{c f_{0} \iint_{A} H d x d y}_{\text {Força Ambiente }}=0 .
$$

Tendo-se em vista a conclusão da seção anterior, ou seja, que todos os vórtices isolados transladam no sentido oeste devido a sua autopropulsão, os parâmetros $c$ e $f_{0}$ presentes na Equação (61) serão sempre negativos.

A força de Coriolis atua sempre no sentido de defletir o movimento para a esquerda no hemisfério Sul, logo, ela será sempre dirigida para sul. 0 segundo termo do lado esquerdo da expressão (61), que corresponde à força de Coriolis, é positivo, 0 que permite a criação das associações positivo/sul e negativo/norte para as três forças que atuam sobre o vórtice isolado em movimento. Na subseção 3.1, dedicada à força $\beta$, foi concluído que esta força aponta para a linha do Equador (ou para norte) no caso de vórtices anticiclônicos. 0 primeiro termo da Equação (61) será negativo neste caso, tendo-se em vista que a função transporte média $\psi$ no interior do vórtice é negativa, o que condiz com a associação negativo/norte. 0 mesmo ocorre com feições ciclônicas, cuja força $\beta$ aponta para sul, conforme mostrou a subseção dedicada à análise desta força. Estes vórtices possuem função transporte média $\psi$ positiva fazendo com que o primeiro termo da Equação (61) seja agora positivo, ou seja, também confirmando a associação positivo/sul feita anteriormente.

0 terceiro termo da Equação (61), com a inclusão do sinal negativo que o precede, será sempre negativo, ou seja, a força ambiente que atua sobre vórtices isolados em movimento no hemisfério Sul será sempre dirigida para a linha do Equador (para norte), o que pode ser comprovado pela Figura 7.

A Figura 11 mostra 0 balanço de forças meridionais para vórtices isolados em movimento no hemisfério Sul. Uma análise similar pode ser feita para vórtices isolados em movimento no hemisfério Norte, a qual é apresentada de forma mais resumida em Nof (1983).

\section{DISCUSSÃO E CONCLUSÃO}

A revisão apresentada neste trabalho mostra que todos os vórtices isolados são autopropulsionados para oeste no plano- $\beta$. Em duas circunstâncias, contudo, estas feições podem não possuir autopropulsão. Uma delas parece ser evidente, ou seja, para $\beta=0$ (desconsideração do plano- $\beta$ ). Porém, esta condição ainda exige uma outra, ou seja, que o fundo oceânico seja plano. A segunda possibilidade, não tão óbvia, ocorre quando a integral da função transporte do vórtice sobre toda a área da feição é nula. Este último caso pode ocorrer com um par de vórtices de circulações opostas [chamados modons, ver Stern (1975)] quando então $\beta \iint_{\psi d x d y}=0$. Outra importante observação é que caso 0 fundo oceânico não seja plano haverá indução de movimento no vórtice devido ao efeito $\beta$ topográfico (e.g., Smith \& O'Brien, 1983), que faz com que os vórtices adquiram movimento no mesmo sentido das ondas topográficas induzidas pela inclinação do fundo. 0 movimento de vórtices isolados sujeitos à ação do parâmetro $\beta$ e sobre fundo não plano estará sujeito à ação cumulativa dos dois efeitos, ou seja, o efeito $\beta$ planetário e 0 efeito $\beta$ topográfico.

0 estudo mostrou ainda que três forças competem no balanço meridional de forças que atuam sobre vórtices isolados em movimento, que são a força $\beta$, a força de Coriolis e a força ambiente. A primeira se deve à diferença do parâmetro de Coriolis entre os hemisférios Norte e Sul do vórtice. A força de Coriolis resulta do efeito da rotação do planeta que tende a defletir movimentos para a esquerda (direita) no hemisfério Sul (Norte) e atua sobre 0 movimento de translação do vórtice. A força ambiente resulta do gradiente de pressão imposto ao vórtice pelo fluido externo deslocado pela feição enquanto em movimento. Esta força é nula para vórtices do tipo lente.

Diversas análises podem ser feitas com relação às forças meridionais que agem sobre vórtices isolados em movimento 


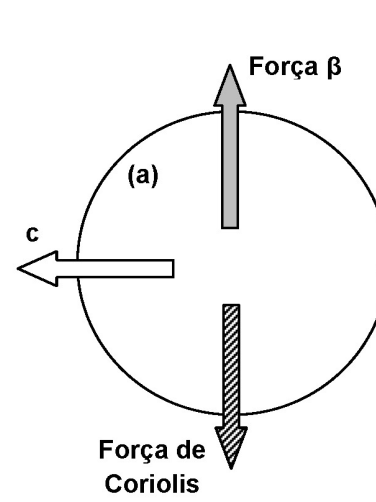

(a) Vórtice anticiclônico do tipo lente

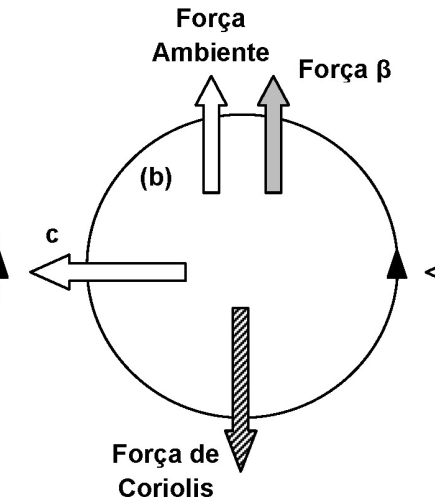

(b) Vórtice anticiclônico do tipo não-lente

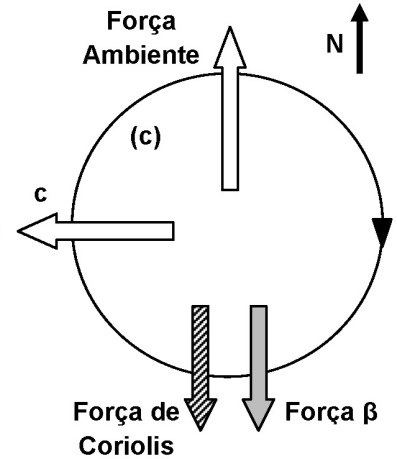

(c) Vórtice ciclônico do tipo não-lente

Figura 11 - Forças meridionais (sem proporção entre si) que agem sobre vórtices isolados em movimento de translação zonal no hemisfério Sul. A seta branca horizontal corresponde à velocidade $c$ dos vórtices que é dirigida para oeste. As setas cinzas correspondem à força $\beta$, as setas hachuradas indicam a força de Coriolis e as setas brancas verticais mostram a força ambiente.

autopropulsionado para oeste. Como exemplo, faremos uma expansão da análise da atuação da força $\beta$ em vórtices ciclônicos e anticiclônicos. Conforme Nof (1983), a velocidade orbital e profundidade de vórtices isolados anticiclônicos, com perfil linear de velocidade, têm como expressão, respectivamente

$$
\begin{aligned}
& v_{\theta}=-R_{0} f_{0} r \quad \mathrm{e} \\
& h=H-R_{0}\left(R_{0}-1\right) f_{0}^{2}\left(r_{0}^{2}-r^{2}\right) / 2 g^{\prime} .
\end{aligned}
$$

Nas expressões (62) $v_{\theta}$ é a velocidade orbital do vórtice, onde $v_{\theta}=v_{\theta}(r)$, a qual é considerada positiva (por convenção) no sentido anti-horário; $r$ é 0 raio da feição para um determinado valor de $v_{\theta}(r) ; r_{0}$ é 0 raio do vórtice medido do centro até a sua periferia; $H$ é a profundidade da feição em $r=$ $r_{0}$ e $R_{0}$ é 0 número de Rossby do vórtice, cuja expressão é dada por $R_{0}=v_{m} / f_{0} r_{m}$, onde $v_{m}$ é a máxima velocidade orbital da feição e $r_{m}$ é 0 raio do vórtice correspondente à máxima velocidade $v_{m}$. A equação da profundidade (62b) é obtida com a aplicação da equação da velocidade orbital (62a) na equação do momentum escrita em coordenadas cilíndricas, para vórtices radialmente simétricos, o que é uma característica destas feições no plano- $f$ (Killworth, 1983), e que é dada por (e.g., Flierl, 1979)

$$
\frac{v_{\theta}^{2}}{r}+f v_{\theta}-g^{\prime} \frac{\partial h}{\partial r}=0 .
$$

A condição de contorno $h=H$ em $r=r_{0}$ também é utilizada na solução de (63).

A Equação (63) possui três termos que estão associados à força centrífuga, força de Coriolis e força do gradiente de pressão (e.g., Neumann \& Pierson Jr., 1966), os quais constam da Equação (63) da esquerda para a direita, respectivamente. A importância do termo centrífugo $v_{\theta}^{2} / r$ está diretamente associada ao número de Rossby $R_{0}$ do vórtice, ou seja, ao grau de não-linearidade da feição. Vórtices lineares $\left(R_{0} \ll 1 \mathrm{e}\right.$, consequentemente, de grandes dimensões) possuem o termo centrífugo muito pequeno, estando então em balanço geostrófico ou quase-geostrófico. Vórtices em que o termo de Coriolis pode ser negligenciado $\left(R_{0} \gg 1\right.$, o que caracteriza vórtices muito pequenos) estão em balanço ciclostrófico, ou seja, a força centrífuga e a força do gradiente de pressão estão em equilíbrio. No caso do balanço gradiente, em que as três forças possuem ordens similares, os vórtices possuem tamanho intermediário e $R_{0}$ de ordem unitária $\left(R_{0} \sim 1\right)$.

A função transporte $\psi$ do vórtice pode ser obtida a partir das expressões (28). Convertendo-se estas expressões para coordenadas cilíndricas, e novamente assumindo-se que a feição é radialmente simétrica, obtém-se

$$
\frac{\partial \psi}{\partial r}=v_{\theta} h .
$$

A utilização das expressões (62) na Equação (64), seguida de integração, com a condição de contorno de que $\psi=0 \mathrm{em}$ $r=r_{0}$, tem como resultado

$$
\begin{gathered}
\psi=\frac{R_{0} f_{0} H\left(r_{0}^{2}-r^{2}\right)}{2} \\
-\frac{R_{0}^{2}\left(R_{0}-1\right) f_{0}^{3}\left(r_{0}^{2}-r^{2}\right)^{2}}{8 g^{\prime}} .
\end{gathered}
$$


0 cálculo da força $\beta$ no vórtice anticiclônico é realizado com a ajuda da expressão

$$
F_{\text {beta }}=\beta \iint_{A} \psi d x d y .
$$

a qual é oriunda da Equação (61). A utilização de (65) na Equação (66) fornece uma expressão para o cálculo da força $\beta$ em vórtices anticiclônicos $F_{\text {beta }(A C)}$, que é dada por

$$
\begin{gathered}
F_{\text {beta }(A C)}=\frac{\pi R_{0} f_{0} H \beta r_{0}^{4}}{4} \\
-\frac{\pi R_{0}^{2}\left(R_{0}-1\right) f_{0}^{3} \beta r_{0}^{6}}{24 g^{\prime}} .
\end{gathered}
$$

Esta expressão será temporariamente deixada de lado enquanto a força $\beta$ dos vórtices ciclônicos será agora analisada. Conforme Nof (1983), a velocidade orbital e profundidade de vórtices isolados ciclônicos, com perfil linear de velocidade, têm como expressão

$$
\begin{aligned}
& v_{\theta}=R_{0} f_{0} r \quad \mathrm{e} \\
& h=H-R_{0}\left(1+R_{0}\right) f_{0}^{2}\left(r_{0}^{2}-r^{2}\right) / 2 g^{\prime} .
\end{aligned}
$$

Raciocínio similar àquele aplicado para os vórtices anticiclônicos conduz a seguinte expressão para a força $\beta$ de feições ciclônicas $F_{\text {beta }(C)}$ :

$$
\begin{gathered}
F_{\text {beta }(C)}=-\frac{\pi R_{0} f_{0} H \beta r_{0}^{4}}{4} \\
+\frac{\pi R_{0}^{2}\left(1+R_{0}\right) f_{0}^{3} \beta r_{0}^{6}}{24 g^{\prime}} .
\end{gathered}
$$

Uma análise simultânea das expressões (67) e (69) será agora realizada. 0 primeiro termo da expressão da força $\beta$ para vórtices anticiclônicos no hemisfério Sul é negativo, conforme mostra a Equação (67). 0 segundo termo desta mesma expressão é aproximadamente nulo para vórtices intermediários e negativo para grandes vórtices. Vórtices anticiclônicos de pequeno tamanho $\left(R_{0} \gg 1\right)$ não podem existir, pois a força de Coriolis, dirigida para dentro da feição, não é capaz de equilibrar as forças centrífuga e a do gradiente de pressão, que são dirigidas para fora da feição, por ser muito pequena. Resumindo, os dois termos da Equação (67) são: (a) negativos (e se somam) para grandes vórtices ou (b) praticamente apenas o primeiro termo (negativo) resulta no caso de vórtices intermediários, pois o segundo termo seria aproximadamente nulo caso $R_{0} \sim 1$. A força $\beta$ de vórtices anticiclônicos aponta para Norte, conforme visto na subseção dedicada a esta força. Pela associação negativo/norte feita anteriormente, a força $\beta$ destes vórtices deve resultar negativa, o que está confirmado pela análise feita na expressão (67).
A força $\beta$ para vórtices ciclônicos é descrita pela Equação (69). Cabe destacar que o primeiro termo desta equação é oposto (positivo) e idêntico ao primeiro termo da Equação (67). 0 segundo termo da expressão (69) é negativo para vórtices pequenos, intermediários e grandes. Resumindo, na situação de vórtices ciclônicos há uma diferença entre os dois termos da equação da força $\beta$, pois eles possuem sinais opostos.

Supondo-se agora o seguinte cenário: dois vórtices de mesmo raio $r_{0}$, com a mesma velocidade periférica $v_{\theta}$ (suposta a máxima velocidade orbital do vórtice), ou seja, com o mesmo número de Rossby $R_{0}$, e mesma profundidade $H$ ao longo de sua borda, porém com circulações opostas. Conforme mostrou a análise anterior suas forças $\beta$ terão dimensões completamente diferentes, sendo esta força maior para a situação de feições anticiclônicas de qualquer dimensão. Como a força $\beta$ é oriunda da diferença líquida do parâmetro de Coriolis entre os hemisférios Norte e Sul do vórtice, esta conclusão pode ser reforçada fazendo-se uma análise agora sobre o volume das duas feições. 0 volume de um vórtice anticiclônico de mesmas características de uma feição ciclônica será sempre maior, resultando numa força líquida interna também maior, o que corrobora com a análise das expressões matemáticas das forças $\beta$ feita acima.

A aplicação das Equações (62) na expressão (57) permite concluir que a velocidade de deriva de vórtices isolados anticiclônicos depende inversamente do tamanho da feição, expresso pelo raio $r_{0}$ da feição, e diretamente da sua amplitude, neste caso considerada como 0 deslocamento $\xi$ da termoclina (ou interface) do vórtice. Com a utilização da expressão (68) em (57) conclui-se também que a velocidade de deriva de vórtices isolados ciclônicos depende diretamente do tamanho da feição e inversamente da sua amplitude (Nof, 1983). A comparação dos dois resultados mostra ainda que vórtices anticiclônicos transladam mais rapidamente que feições ciclônicas de mesma profundidade externa $H$, mesma amplitude $\xi$ e mesmo tamanho ( $r_{0}$ iguais), conforme mostra Nof (1983). Estes comparativos mostram que 0 comportamento dinâmico de vórtices isolados, com circulações opostas, é completamente diferente.

A autopropulsão de vórtices isolados no sentido oeste sofre desvios que afastam estas feições de um movimento puramente zonal. Estes desvios estão diretamente associados com as nãolinearidades das feições (Firing \& Beardsley, 1976), ocorrendo no sentido dos pólos para vórtices ciclônicos e no sentido da linha do Equador para vórtices anticiclônicos. Cabe destacar que o desvio da trajetória de vórtices isolados com relação ao movimento puramente zonal, também pode se dever à ação de correntes externas ou à presença de outros vórtices próximos. 
A aproximação e a interação entre um vórtice e um obstáculo com orientação meridional promovem 0 surgimento de outras forças meridionais, as quais estão associadas com 0 chamado efeito imagem e com o vazamento de líquido do interior da feição (e.g., Nof, 1988ab, 1999). Contudo, estas forças não são o escopo deste estudo.

\section{AGRADECIMENTOS}

Este trabalho é uma contribuição ao projeto SOS-CLIMATE (CNPq/MCT/PROANTAR, processo: 520189/2006-0), o qual recebe apoio do Conselho Nacional de Desenvolvimento Científico e Tecnológico (CNPq), Ministério da Ciência e Tecnologia (MCT) e do Programa Antártico Brasileiro (PROANTAR). É também uma contribuição ao projeto MOVAR (CNPq/Universal-2008, processo: 474057/2009-9), o qual recebe apoio do CNPq. José L.L. Azevedo agradece 0 apoio da Coordenação de Aperfeiçoamento de Pessoal de Nível Superior (CAPES, BEX 2097.07-9) e do InterAmerican Institute for Global Change Research IAI/CRN 2076, 0 qual tem apoio da US National Science Foundation (NSF Grant GR0-0452325). Mauricio M. Mata agradece 0 apoio do CNPq (Pq-2 processo 301623/2006-6). Os autores também gostariam de manifestar seus agradecimentos a Doron Nof (Florida State University - FSU), pelas sugestões que muito contribuíram na elaboração deste estudo, e também aos revisores anônimos que colaboraram com este trabalho.

\section{REFERÊNCIAS}

BATCHELOR GK. 1998. An Introduction to Fluid Dynamics. Cambridge University Press, Cambridge, 17 ed., 620 p.

BYRNE DA. 2000. From the Agulhas to the South Atlantic: Measuring Inter-Ocean Fluxes. Tese de Doutorado, Columbia University, 181 p.

BYRNE DA, GORDON AL \& HAXBY WF. 1995. Agulhas Eddies: A Synoptic View Using Geosat ERM Data. Journal of Physical Oceanography, 25: 902-917.

CHELTON DB, SCHLAX MG, SAMELSON RM \& SZOEKE RA. 2007. Global Observations of Large Oceanic Eddies. Geophysical Research Letters, 34, L15606, doi: 10.1029/2007GL030812.

CUSHMAN-ROISIN B. 1994. Introduction to Geophysical Fluid Dynamics. Prentice Hall, $320 p$.

CUSHMAN-ROISIN B, CHASSIGNET EP \& TANG B. 1990. Westward Motion of Mesoscale Eddies. Journal of Physical Oceanography, 20: 758-768.

FIRING E \& BEARDSLEY RC. 1976. The Behavior of a Barotropic Eddy on a $\beta$-Plane. Journal of Physical Oceanography, 6: 57-65.
FLIERL GR. 1979. A Simple Model for a Structure of Warm and Cold Core Rings. Journal of Geophysical Research, 84(C2): 781-785.

FOX RW \& McDONALD AT. 1978. Introdução à Mecânica dos Fluidos. Editora Guanabara Dois, 2 ed., 562 p.

GILL A. 1982. Atmosphere-Ocean Dynamics. International Geophysics Series - Volume 30, Academic Press, $684 p$

HWANG C, WU C-R \& KAO R. 2004. TOPEX/Poseidon Observations of Mesoscale Eddies over the Subtropical Countercurrent: Kinematic Characteristics of an Anticyclonic Eddy and a Cyclonic Eddy. Journal of Geophysical Research, 109(C08013), doi: 10.1029/2003JC002026.

KILLWORTHPD. 1983. On the Motion of Isolated Lenses on a Beta-Plane. Journal of Physical Oceanography, 13: 368-376.

KUNDU PK \& COHEN IM. 2008. Fluid Mechanics. Academic Press, Elsevier, 4 ed., $878 p$.

LENTINI C, GONI G \& OLSON D. 2006. Investigation of Brazil Current Rings in the Confluence Region. Journal of Geophysical Research 111(C06013), doi: 10.1029/2005JC002988.

MILNE-THOMSON LM. 1968. Theoretical Hydrodynamics. Dover Publications, $744 p$

NEUMANN G \& PIERSON JR WJ. 1966. Principles of Physical Oceanography. Prentice-Hall Inc., Englewood Cliffs, N.J., 550 p.

NOF D. 1981a. On the Dynamics of Equatorial Outflows with Application to the Amazon's Basin. Journal of Marine Research, 39(1): 1-29.

NOF D. 1981b. On the $\beta$-Induced Movement of Isolated Baroclinic Eddies. Journal of Physical Oceanography, 11: 1662-1672.

NOF D. 1983. On the Migration of Isolated Eddies with Application to Gulf Stream Rings. Journal of Marine Research, 41: 399-425.

NOF D. 1988a. Draining Vortices. Geophys. Astrophys. Fluid Dyn., 42: 187-208.

NOF D. 1988b. Eddy-wall Interactions. Journal of Marine Research, 46 $527-555$

NOF D. 1999. Strange Encounters of Eddies with Walls. Journal of Marine Research, 57: 739-761.

OLSON DB. 1991. Rings in the Ocean. Annu. Rev. Earth Planet. Sci., 19: 283-311.

PEDLOSKY J. 1987. Geophysical Fluid Dynamics. Ed. Springer-Verlag, New York, 2 ed., $710 \mathrm{p}$.

ROBINSON AR. 1983. Eddies in Marine Science. Springer-Verlag, 616 p.

SHI C \& NOF D. 1994. The Destruction of Lenses and Generation of Wodons. Journal of Physical Oceanography, 24: 1120-1136.

SMITH DC \& O'BRIEN JJ. 1983. The Interaction of a Two-Layer Isolated Mesoscale Eddy With Bottom Topography. Journal of Physical Oceanography, 13: 1681-1697. 
SOUZA RB. 2005. Oceanografia por Satélites. Editora Oficina do Texto, São Paulo (SP), Brasil, 336 p.

SOUZA RB, MATA MM, GARCIA CAE, KAMPEL M, OLIVEIRA EN \& LORENZZETTI JA. 2006. Multi-sensor Satellite and In Situ Measure- ments of a Warm Core Ocean Eddy South of the Brazil-Malvinas Confluence Region. Remote Sensing of Environment, 100: 52-66.

STERN ME. 1975. Minimal Properties of Planetary Eddies. Journal of Marine Research, 33: 1-13.

\section{NOTAS SOBRE OS AUTORES}

José Luiz Lima de Azevedo possui graduação em Engenharia Química (FURG, 1982), Mestrado em Engenharia Elétrica (UFSC, 1996) e Doutorado em Oceanografia Física, Química e Geológica (FURG, 2009), tendo realizado um período de estudos (2008) na Florida State University. É professor da FURG desde 1984, estando atualmente lotado no Instituto de Oceanografia. Estuda o encontro de vórtices anticiclônicos com uma borda continental, com ênfase na interação entre os vórtices provenientes da Corrente das Agulhas com a borda continental brasileira e sua corrente de contorno associada, a Corrente do Brasil. Tem ainda como área de interesse a modelagem numérica, com a utilização do POM e do modelo isopicnal Bleck \& Boudra.

Mauricio Magalhães Mata é graduado em Oceanografia (FURG, 1991) e Mestre em Sensoriamento Remoto (UFRGS, 1996). Em 2001 tornou-se Doutor em Oceanografia Física pela Flinders University of South Australia, Adelaide, Austrália. Desde 1992 é Professor Permanente da FURG, estando atualmente lotado no Instituto de Oceanografia. Sua linha de pesquisa concentra-se na circulação oceânica de meso e larga-escala, com ênfase em correntes de contorno oeste e Oceanografia da Antártica. Tem especial interesse nos processos interanuais que controlam a formação e exportação de águas de fundo no entorno do continente antártico. 